\title{
Neoadjuvant Chemotherapy Reduces Adjuvant Radiation Therapy in Patients with Locally Advanced Cervical Cancer
}

\author{
Yuhui Huang \\ Wuhan Union Hospital \\ Lei Chen \\ Wuhan Union Hospital \\ Jing Cai \\ Wuhan Union Hospital \\ Lu Yang \\ Wuhan Union Hospital \\ Si Sun \\ Wuhan Union Hospital \\ Jing Zhao \\ Wuhan Union Hospital \\ Zhoufang Xiong \\ Wuhan Union Hospital \\ Zehua Wang ( $\nabla$ zehuawang@163.net) \\ Wuhan Union Hospital https://orcid.org/0000-0001-6423-8219
}

\section{Research}

Keywords: Neoadjuvant chemotherapy, Locally advanced cervical cancer, Adjuvant radiotherapy,

Prognosis

Posted Date: September 27th, 2021

DOI: https://doi.org/10.21203/rs.3.rs-915571/v1

License: (c) (i) This work is licensed under a Creative Commons Attribution 4.0 International License.

Read Full License 


\section{Abstract}

Background: To investigated whether carboplatin-paclitaxel neoadjuvant chemotherapy (NACT) benefits patients with locally advanced cervical cancer (LACC) through avoiding or delaying postoperative radiation.

Methods: Patients with International Federation of Gynecology and Obstetrics (FIGO 2009) stage IB2-IIA2 who received carboplatin-paclitaxel chemotherapy followed by radical surgery (NACT group) or received primary radical surgery (PRS group) between 2007 and 2017 at our hospital were included. Their clinicopathological characteristics, treatments and follow-up data were retrospectively collected and analyzed. The clinical outcomes, including adjuvant radiation, progression-free survival (PFS), and overall survival (OS), were compared between the groups.

Results: There were 197 and 217 patients included in the NACT group and PRS group, respectively. The baseline characteristics were significantly different between the groups, with more patients with more advanced tumor stages and larger tumor sizes in the NACT group. Postoperative pathology examination revealed lower incidences of deep stromal invasion in the NACT group than in the PRS group. More importantly, the cumulative postoperative radiation rate was significantly lower in the NACT group ( $P=$ 0.041), while the differences in 5-year OS and PFS were not statistically significant between the groups.

Conclusions: NACT reduces the pathological risk factors and adjuvant radiation without compromising survival in patients with LACC, which may protect younger patients from radiation-related side effects and subsequently improve the quality of life.

\section{Background}

Cervical cancer is a major cause of cancer mortality in women worldwide. According to global cancer statistics, there were 604,000 new cases and 342,000 cervical cancer-related deaths in 2020 (1). While radical surgery is accepted as the standard treatment for early-stage cervical cancer, the optimal treatment for patients with stage IB2-IIA2 remains controversial.

Though concurrent chemoradiation is recommended as the standard treatment (2), it sometimes brings serious complications, such as bladder dysfunction, sexual dysfunction and gastrointestinal dysfunction. Therefore, how to reduce the need for radiotherapy to improve the quality of patients' life is still a challenge. Neoadjuvant chemotherapy (NACT) followed by radical surgery has been widely used for patients with locally advanced cervical cancer (LACC) to reduce tumor volume, improve the rate of resection and control the potential micro-metastasis. Previous studies have also shown that NACT may have an impact on the clinicopathological risk factors and postoperative radiotherapy (3-5). One phase III trial reported that NACT with BOMP (bleomycin, vincristine, mitomycin and cisplatin) regimen before radical surgery significantly reduced both the proportion of patients who met the criteria for postoperative radiation and the proportion of patients who received postoperative radiation compared to primary radical surgery (PRS) (6). Similar results were also reported in another multicenter study with NACT 
consisting of IP (irinotecan plus cisplatin) and TP (paclitaxel plus cisplatin) regimens (3). However, Lei Li et al. failed to identify these findings in their study with NACT of TC (paclitaxel plus carboplatin), TP and PF (fluorouracil plus cisplatin) regimens (7), as well as another study with four different chemotherapy regimens (8). In these studies, the NACT regimens were different and the response rates varies from $58.8-75.7 \%$, the inconsistent results impacted the credibility of the effect of NACT on postoperative radiotherapy. Therefore, the potential benefits of different regimens of NACT on postoperative adjuvant radiotherapy need to be tested further.

There have been no study investigating the effect of NACT with carboplatin-paclitaxel on adjuvant radiation, which has been recognized as one of the most effective regimens with a high response rate of $95 \%$ and mild toxicity (9). Thus, we performed this retrospective study to investigate whether NACT with paclitaxel and carboplatin reduces the clinicopathological risk factors and adjuvant radiation in patients with LACC, and compare the oncologic outcomes between patients underwent NACT followed by radical surgery and patients underwent PRS.

\section{Methods}

\section{Patients}

All patients with International Federation of Gynecology and Obstetrics (FIGO 2009) stage IB2-IIA2 cervical cancer who underwent carboplatin-paclitaxel chemotherapy followed by radical hysterectomy and pelvic lymphadenectomy (NACT group) or underwent primary radical hysterectomy and pelvic lymphadenectomy (PRS group) between 2007 and 2017 were reviewed. All patients were diagnosed by cervical biopsy and pathology before treatment, and the staging was based on pelvic examination, Magnetic Resonance Imaging (MRI) and Computed Tomography (CT). Patients who did not complete the radical surgery, those who received preoperative radiotherapy and those who had missing data were excluded.

Clinical and pathological information including age, FIGO stage, tumor size, histology, differentiation, lymph vascular space invasion (LVSI), parametrial involvement, deep stromal invasion, surgical margin and lymph node status were collected from patients' medical records.

The study was approved by the Ethics Committee of the Tongji Medical College, Huazhong University of Science and Technology (No. 2018S452).

\section{Management}

All patients underwent radical hysterectomy and pelvic lymphadenectomy. For the NACT group, carboplatin-paclitaxel regimen was used. Patients intravenously received paclitaxel (paclitaxel liposome for injection, Luye Pharma Group Ltd., Nanjing, China) at $135-175 \mathrm{mg} / \mathrm{m}^{2}$ on the first day and carboplatin (carboplatin for injection, Qilu Phama Ltd., China) at an area under curve (AUC) $=5$ on the 
second day every 3 weeks, the number of NACT cycles ranged from 2 to 3 . Assessment of the response to NACT was based on the Response Evaluation Criteria in Solid Tumors (RECIST, version 1.1) (10). Complete response (CR) was defined as complete disappearance of all target lesions; partial response (PR) was defined as $a \geq 30 \%$ decrease in the sum of the diameters of target lesions; progressive disease (PD) was defined as $a \geq 20 \%$ increase in the sum of the diameters of target lesions or the appearance of new target lesions; stable disease (SD) was defined as the status between PR and PD. The NACT responders (NACT-R) was defined as patients with $C R$ or PR, while the non-responders (NACT-NR) was defined as patients with SD or PD. Deep stromal invasion was defined as $\geq 1 / 3$ cervical stromal invasion. After radical surgery, adjuvant therapy was recommended if patients exhibit any of the high risk factors of lymph node metastasis, parametrial involvement or positive surgical margins, and in patients with 2 or more intermediate risk factors including LVSI, deep stromal invasion, and tumor size $\geq 2 \mathrm{~cm}$.

\section{Follow Up}

The follow up was performed according to the National Comprehensive Center Network (NCCN) (2). The items mainly included gynecological examination, vaginal stump cytological examination, pelvic ultrasound and chest X-ray. The overall survival (OS) was defined as the time from completion of operation to death or to the date of last contact. The Progression-free survival (PFS) was defined as the time from completion of operation to the first appearance of progressive disease or to the date of last contact.

\section{Statistical analysis}

SPSS 23.0 (IBM Corp., Armonk, NY, USA) was used for statistical analysis. The statistical significance of variables was assessed with $\chi 2$ test or Fisher's exact test as appropriate. The survival curves and cumulative radiation curves were performed using the Kaplan-Meier method with GraphPad Prism 7.0 (GraphPad Software, Inc., San Diego, CA, USA), and log-rank test was used to assess the significance of the differences. P-value $<0.05$ was considered statistically significant.

\section{Results}

\section{The characteristics of the patients}

A total of 414 patients with stage IB2-IIA2 disease were included in this study. 197 patients received NACT followed by radical surgery, 217 patients received PRS. Their characteristics are listed in Table 1. The patients in the two groups were comparable in age, histological type and tumor differentiation, but the patients in the NACT group had more advanced tumor stages and larger tumor sizes. At the end of NACT, the total response rate was $62.4 \%$, of which 34 (17.3\%) patients achieved CR and $89(45.2 \%)$ patients achieved PR. The NACT non-responders included 71 (36.0\%) patients with SD and $3(1.5 \%)$ patients with $\mathrm{PD}$. 
Table 1

Characteristics of the patients with locally advanced cervical cancer

\begin{tabular}{|c|c|c|c|}
\hline Characteristics & NACT & PRS & P-value \\
\hline & $\mathrm{N}(\%)$ & $\mathrm{N}(\%)$ & ( $\chi 2$ test) \\
\hline Total & 197 & 217 & \\
\hline Age (year) & & & 0.051 \\
\hline$<45$ & $79(40.1)$ & $67(30.9)$ & \\
\hline$\geq 45$ & 118 (59.9) & $150(69.1)$ & \\
\hline Stage (FIGO 2009) & & & $<0.001$ \\
\hline IB2 & $88(44.7)$ & $33(15.2)$ & \\
\hline IIA1 & $17(8.6)$ & $166(76.5)$ & \\
\hline IIA2 & $92(46.7)$ & $18(8.3)$ & \\
\hline Primary tumor size $(\mathrm{cm})$ & & & $<0.001$ \\
\hline$<4.0$ & $14(7.1)$ & $149(68.7)$ & \\
\hline $4.0 \sim 4.9$ & $91(46.2)$ & $42(19.4)$ & \\
\hline $5.0 \sim 5.9$ & $59(29.9)$ & $23(10.6)$ & \\
\hline$\geq 6.0$ & $33(16.8)$ & $3(1.4)$ & \\
\hline Histology & & & 0.356 \\
\hline Squamous & $168(85.3)$ & $177(81.6)$ & \\
\hline Non-squamous & $29(14.7)$ & $40(18.4)$ & \\
\hline Histologic grade & & & 0.906 \\
\hline G1 & $17(8.6)$ & $17(7.8)$ & \\
\hline G2 & $101(51.3)$ & $116(53.5)$ & \\
\hline G3 & $56(28.4)$ & $56(25.8)$ & \\
\hline Unknown & 23 (11.7) & $28(12.9)$ & \\
\hline
\end{tabular}

\section{Neoadjuvant Chemotherapy Reduced Risk Factors And Adjuvant Radiation}


To investigate whether NACT could reduce the risk factors associated with recurrence and death that are prerequisites for adjuvant radiotherapy, we compared the clinicopathological risk factors between the two groups. As shown in Table 2, after NACT, the tumor volume in the NACT group significantly shrank, and there were more tumors $\leq 2.0 \mathrm{~cm}$ in the NACT group than in the PRS group $(36.0 \%$ vs $19.8 \%, P<0.001)$. The postoperative pathology examination also revealed a lower incidence of deep stromal invasion in the NACT group than in the PRS group $(46.7 \%$ vs $59.5 \%, P=0.023)$, whereas the two groups showed no significant differences in parametrial involvement, lymph node metastasis, LVSI and positive surgical margin. 
Table 2

The clinicopathological risk factors in patients with locally advanced cervical cancer

\begin{tabular}{|c|c|c|c|}
\hline Varaibles & NACT & PRS & P-value \\
\hline & $N(\%)$ & $N(\%)$ & x2 test \\
\hline Total & 197 & 217 & \\
\hline Preoperative tumor size & & & $<0.001$ \\
\hline$\leq 2.0 \mathrm{~cm}$ & $71(36.0)$ & $43(19.8)$ & \\
\hline $2.1 \sim 4.0 \mathrm{~cm}$ & 78 (39.6) & $126(58.1)$ & \\
\hline$\varangle 4.0 \mathrm{~cm}$ & $48(24.4)$ & $48(22.1)$ & \\
\hline Parametrial involvement & & & 0.776 \\
\hline Negative & $169(85.8)$ & $184(84.8)$ & \\
\hline Positive & $28(14.2)$ & $33(15.2)$ & \\
\hline Lymph node metastasis & & & 0.336 \\
\hline Negative & $134(68.0)$ & $157(72.4)$ & \\
\hline Positive & $63(32.0)$ & $60(27.6)$ & \\
\hline LVSI & & & 0.135 \\
\hline Negative & $150(76.1)$ & $151(69.6)$ & \\
\hline Positive & $47(23.9)$ & $66(30.4)$ & \\
\hline Cervical stromal invasion & & & 0.023 \\
\hline$<1 / 3$ & $40(20.3)$ & $36(16.6)$ & \\
\hline $1 / 3 \sim 2 / 3$ & $20(10.2)$ & $41(18.9)$ & \\
\hline$\geq 2 / 3$ & $72(36.5)$ & $88(40.6)$ & \\
\hline Unknown & $65(33.0)$ & $52(24.0)$ & \\
\hline Surgery margin & & & 0.128 \\
\hline Negative & $190(96.4)$ & $202(93.1)$ & \\
\hline Positive & $7(3.6)$ & $15(6.9)$ & \\
\hline
\end{tabular}

Furthermore, we compared the cumulative postoperative radiation rates in different groups to determine whether NACT reduced the adjuvant radiation in patients with LACC. As shown in Fig. 1A, the cumulative radiation rate of the NACT group was significantly lower than that of the PRS group $(54.7 \%$ vs $65.1 \%$; $P=$ 
0.041). In the subgroup analysis (Fig. 1B), the cumulative radiation rate of the NACT responders was significantly lower than that of the PRS group $(54.2 \%$ vs $65.1 \% ; P=0.013)$, but there was no significant difference between the non-responders and PRS group (58.8\% vs $65.1 \% ; P=0.695)$.

\section{Survival}

We then compared the OS and PFS rates between the two groups to investigate whether NACT impacts the survival of patients with LACC. The median follow-up time was 45 (range, $3 \sim 150$ ) months. The 5year OS (78.3\% vs $83.0 \%, P=0.306)$ (Fig. 1C) and PFS (64.5\% vs 70.6\%, $P=0.207$ ) (Fig. 1D) of the NACT group were comparable with those in the PRS group, suggesting that NACT did not compromise the survival.

\section{Discussion}

As the age at which women develop cervical cancer is decreasing and radiation may have serious implications that reduce the quality of patients' life, reducing radiation is an important challenge for patients with LACC. In this study, our results revealed that NACT with paclitaxel and carboplatin significantly reduced the clinicopathological risk factors and cumulative adjuvant radiation rate when compared to the PRS group, without compromising the survival. These findings may provide important help in the management of LACC to improve the quality of life.

Though concurrent chemoradiation is recommended as the standard treatment for patients with LACC, as the age at which women develop cervical cancer is decreasing, treatment that protects physiological function and improves quality of life is important, and radical surgery has been chosen for LACC. However, after radical surgery, some patients still present with pelvic lymph node metastasis, parametrial involvement, positive surgery margin, LVSI and deep stromal invasion, which are identified as risk factors for recurrence and death $(11,12)$, and adjuvant radiation is recommended if patients exhibit these risk factors. These patients are then faced with increased treatment and complications.

In an effort to improve the prognosis and quality of life of patients with LACC, NACT followed by radical surgery has been proposed as a promising strategy for LACC (13), which was believed to be able to reduce the risk factors of recurrence and death, and reduce the need for postoperative adjuvant radiotherapy. According to a randomized study, the pelvic lymph node metastasis ( $25.0 \%$ vs $42.9 \%, P=$ $0.025)$ and parametrial infiltration $(25.0 \%$ vs $41.4 \%, P=0.038)$ rates were significantly lower in the NACT (with cisplatin, mitomycin and 5-fluorouracil) group than in the PRS group (14). Yang et al. reported in their multicenter study that the incidence of LVSI and deep stromal invasion were both significantly reduced after NACT with IP and TP when compared to the PRS group (4.7\% vs $18.2 \%, P=0.002 ; 45.8 \%$ vs $68.2 \%, P=0.001$ ) (3), and Kim et al. also obtained similar results in their study with NACT consisting of paclitaxel/carboplatin, 5-fluorouracil/cisplatin and 5-fluorouracil/carboplatin (15). In this study, we observed that the rates of patients with tumor $>2 \mathrm{~cm}$ and deep stromal invasion in the NACT group were 
significantly lower than those in the PRS group, while there were more patients with more advanced stages and larger primary tumor sizes in the NACT group.

Regarding postoperative radiation, our results showed that the cumulative radiation rate was significantly reduced in the NACT group compared to the PRS group ( $54.7 \%$ vs $65.1 \% ; P=0.041)$, especially for the responders. Similarly, another study with NACT consisting of paclitaxel and cisplatin/carboplatin also reported that the adjuvant radiotherapy was administered to less patients in NACT group compared to PRS group (13). Katsumata $\mathrm{N}$ revealed that the proportion of patients who met the criteria for postoperative radiation ( $72 \%$ vs $89 \%, P=0.015)$ and patients who received postoperative radiation (58\% vs $79 \%, P=0.015$ ) were both significantly lower in the NACT group than in the PRS group (6). Yang et al also reported that the rates of postoperative adjuvant radiotherapy and chemoradiation in the NACT group were lower than that of the PRS group, though without significant difference (3).

While the short-term efficacy of NACT is certain, whether NACT affects locally advanced cervical cancer patients with long-term survival remains controversial $(16,17)$. Yin et al. performed a retrospective study to compare the long-term survival of NACT followed by radical surgery and primary radical surgery, and the results showed that the NACT group had significantly higher PFS (HR $=1.870, P=0.0031)$ and OS $(H R=1.813, P=0.0175)$ rates than the PRS group (16). However, there were also studies that failed to obtain similar results. A phase III trial was conducted to determine whether NACT impacts the survival of LACC, and it finally failed to find any benefit of NACT group, with similar PFS rates ( $56.2 \%$ vs $53.8 \%$ ) and OS rates (63.3\% vs $60.7 \%$ ) in the NACT group compared to the PRS group (18), which was in consistence with our previous study (19). In the present study, the 5-year OS and PFS of the NACT group $(78.3 \%$ and $64.5 \%$ ) were similar to those of the PRS group (83.0\% and $70.6 \%$ ). All these results indicated that NACT may be useful for improving the survival of patients with LACC or may confer comparable survival, it at least did not worsen the long-term survival.

Our study clarified the impact of carboplatin-paclitaxel NACT on postoperative risk factors and cumulative radiotherapy in patients with LACC. However, it had several limitations. First, it was a singlecenter retrospective study in which selection bias and confounding bias were inevitable; second, the patients in NACT group and PRS group were not very well matched; third, in terms of OS and PFS, we did not make comparisons to concurrent chemoradiation, which is also effective against LACC. Thus, further multicenter and randomized trials are need to verify the effect of NACT on adjuvant radiation.

\section{Conclusions}

In conclusion, our results suggest that carboplatin-paclitaxel NACT before RS can reduce the rate of patients who received postoperative radiotherapy, without compromising the survival.

\section{Abbreviations}

Neoadjuvant chemotherapy (NACT) 
Locally advanced cervical cancer (LACC)

Progression-free survival (PFS)

Overall survival (OS)

Primary radical surgery, PRS

Bleomycin, vincristine, mitomycin and cisplatin regimen, BOMP

Irinotecan plus cisplatin regimen, IP

Paclitaxel plus cisplatin regimen, TP

Fluorouracil plus cisplatin, PF

Magnetic Resonance Imaging, MRI

Computed Tomography, CT

Lymph vascular space invasion, LVSI

Area under curve, AUC

Complete response, $\mathrm{CR}$

Partial response, PR

Progressive disease, PD

Stable disease, SD

NACT responders, NACT-R

NACT non-responders, NACT-NR)

National Comprehensive Center Network, NCCN

\section{Declarations}

\section{Ethics approval and consent to participate}

This study was approved by the Ethics Committee of the Tongji Medical College, Huazhong University of Science and Technology (No. 2018S452).

Consent for publication 
Not applicable

\section{Availability of data and materials}

Data available within the article or its supplementary materials.

\section{Competing interests}

The authors have no conflicts of interest to declare.

\section{Funding}

This study was supported by the National Natural Science Foundation of China (81974413) and the Natural Science Foundation of Hubei Province, China (2019CFB704).

\section{Authors' contributions}

Conception \& Design of Study: Zehua Wang and Zhoufang Xiong

Data Collection: Yuhui Huang, Lei Chen, Lu Yang and Jing Zhao

Data Analysis \& Interpretation : Jing Cai and Si Sun

Responsible Surgeon : Lu Yang, Zhoufang Xiong and Zehua Wang

Statistical Analysis: Yuhui Huang and Jing Zhao

Manuscript Preparation: Yuhui Huang and Lei Chen

\section{Acknowledgements}

We thanked Dr. Qinghui Zhou for her help of data collection.

\section{References}

1. Sung H, Ferlay J, Siegel RL, Laversanne M, Soerjomataram I, Jemal A, et al. Global Cancer Statistics 2020: GLOBOCAN Estimates of Incidence and Mortality Worldwide for 36 Cancers in 185 Countries. CA Cancer J Clin. 2021;71(3):209-49.

2. Koh WJ, Abu-Rustum NR, Bean S, Bradley K, Campos SM, Cho KR, et al. Cervical Cancer, Version 3.2019, NCCN Clinical Practice Guidelines in Oncology. J Natl Compr Canc Netw. 2019;17(1):64-84.

3. Yang Z, Chen D, Zhang J, Yao D, Gao K, Wang H, et al. The efficacy and safety of neoadjuvant chemotherapy in the treatment of locally advanced cervical cancer: A randomized multicenter study. Gynecol Oncol. 2016;141(2):231-9.

4. Rydzewska L, Tierney J, Vale CL, Symonds PR. Neoadjuvant chemotherapy plus surgery versus surgery for cervical cancer. Cochrane Database Syst Rev. 2010(1):CD007406. 
5. Kim HS, Sardi JE, Katsumata N, Ryu HS, Nam JH, Chung HH, et al. Efficacy of neoadjuvant chemotherapy in patients with FIGO stage IB1 to IIA cervical cancer: an international collaborative meta-analysis. Eur J Surg Oncol. 2013;39(2):115-24.

6. Katsumata N, Yoshikawa H, Kobayashi H, Saito T, Kuzuya K, Nakanishi T, et al. Phase III randomised controlled trial of neoadjuvant chemotherapy plus radical surgery vs radical surgery alone for stages IB2, IIA2, and IIB cervical cancer: a Japan Clinical Oncology Group trial (JCOG 0102). Br J Cancer. 2013;108(10):1957-63.

7. Li L, Wu M, Ma S, Tan X, Zhong S. Neoadjuvant chemotherapy followed by radical hysterectomy for stage IB2-to-IIB cervical cancer: a retrospective cohort study. Int J Clin Oncol. 2019;24(11):1440-8.

8. Zhao H, He Y, Zhu LR, Wang JL, Guo HY, Xu T, et al. Effect of neoadjuvant chemotherapy followed by radical surgery for FIGO stage IB2/IIA2 cervical cancer: A multi-center retrospective clinical study. Medicine. 2019;98(21):e15604.

9. Duenas-Gonzalez A, Lopez-Graniel C, Gonzalez-Enciso A, Cetina L, Rivera L, Mariscal I, et al. A phase II study of multimodality treatment for locally advanced cervical cancer: neoadjuvant carboplatin and paclitaxel followed by radical hysterectomy and adjuvant cisplatin chemoradiation. Ann Oncol. 2003;14(8):1278-84.

10. Eisenhauer EA, Therasse P, Bogaerts J, Schwartz LH, Sargent D, Ford R, et al. New response evaluation criteria in solid tumours: revised RECIST guideline (version 1.1). Eur $\mathrm{J}$ Cancer. 2009;45(2):228-47.

11. Gong L, Zhang JW, Yin RT, Wang P, Liu H, Zheng Y, et al. Safety and Efficacy of Neoadjuvant Chemotherapy Followed by Radical Surgery Versus Radical Surgery Alone in Locally Advanced Cervical Cancer Patients. Int J Gynecol Cancer. 2016;26(4):722-8.

12. Gadducci A, Teti G, Barsotti C, Tana R, Fanucchi A, Orlandini C, et al. Clinicopathological variables predictive of clinical outcome in patients with FIGO stage Ib2-llb cervical cancer treated with cisplatin-based neoadjuvant chemotherapy followed by radical hysterectomy. Anticancer Res. 2010;30(1):201-8.

13. Cho YH, Kim DY, Kim JH, Kim YM, Kim YT, Nam JH. Comparative study of neoadjuvant chemotherapy before radical hysterectomy and radical surgery alone in stage IB2-IIA bulky cervical cancer. J Gynecol Oncol. 2009;20(1):22-7.

14. Chen H, Liang C, Zhang L, Huang S, Wu X. Clinical efficacy of modified preoperative neoadjuvant chemotherapy in the treatment of locally advanced (stage IB2 to IIB) cervical cancer: randomized study. Gynecol Oncol. 2008;110(3):308-15.

15. Kim HS, Kim JY, Park NH, Kim K, Chung HH, Kim YB, et al. Matched-case comparison for the efficacy of neoadjuvant chemotherapy before surgery in FIGO stage IB1-IIA cervical cancer. Gynecol Oncol. 2010;119(2):217-24.

16. Yin M, Zhao F, Lou G, Zhang H, Sun M, Li C, et al. The long-term efficacy of neoadjuvant chemotherapy followed by radical hysterectomy compared with radical surgery alone or concurrent 
chemoradiotherapy on locally advanced-stage cervical cancer. Int J Gynecol Cancer. 2011;21(1):929.

17. Yan W, Si L, Ding Y, Qiu S, Zhang Q, Liu L. Neoadjuvant chemotherapy does not improve the prognosis and lymph node metastasis rate of locally advanced cervical squamous cell carcinoma: A retrospective cohort study in China. Medicine. 2019;98(39):e17234.

18. Eddy GL, Bundy BN, Creasman WT, Spirtos NM, Mannel RS, Hannigan E, et al. Treatment of ("bulky") stage IB cervical cancer with or without neoadjuvant vincristine and cisplatin prior to radical hysterectomy and pelvic/para-aortic lymphadenectomy: a phase III trial of the gynecologic oncology group. Gynecol Oncol. 2007;106(2):362-9.

19. Yang L, Guo J, Shen Y, Cai J, Xiong Z, Dong W, et al. Clinical efficacy and safety of paclitaxel plus carboplatin as neoadjuvant chemotherapy prior to radical hysterectomy and pelvic lymphadenectomy for Stage IB2-IIB cervical cancer. Int J Clin Exp Med. 2015;8(8):13690-8.

\section{Figures}
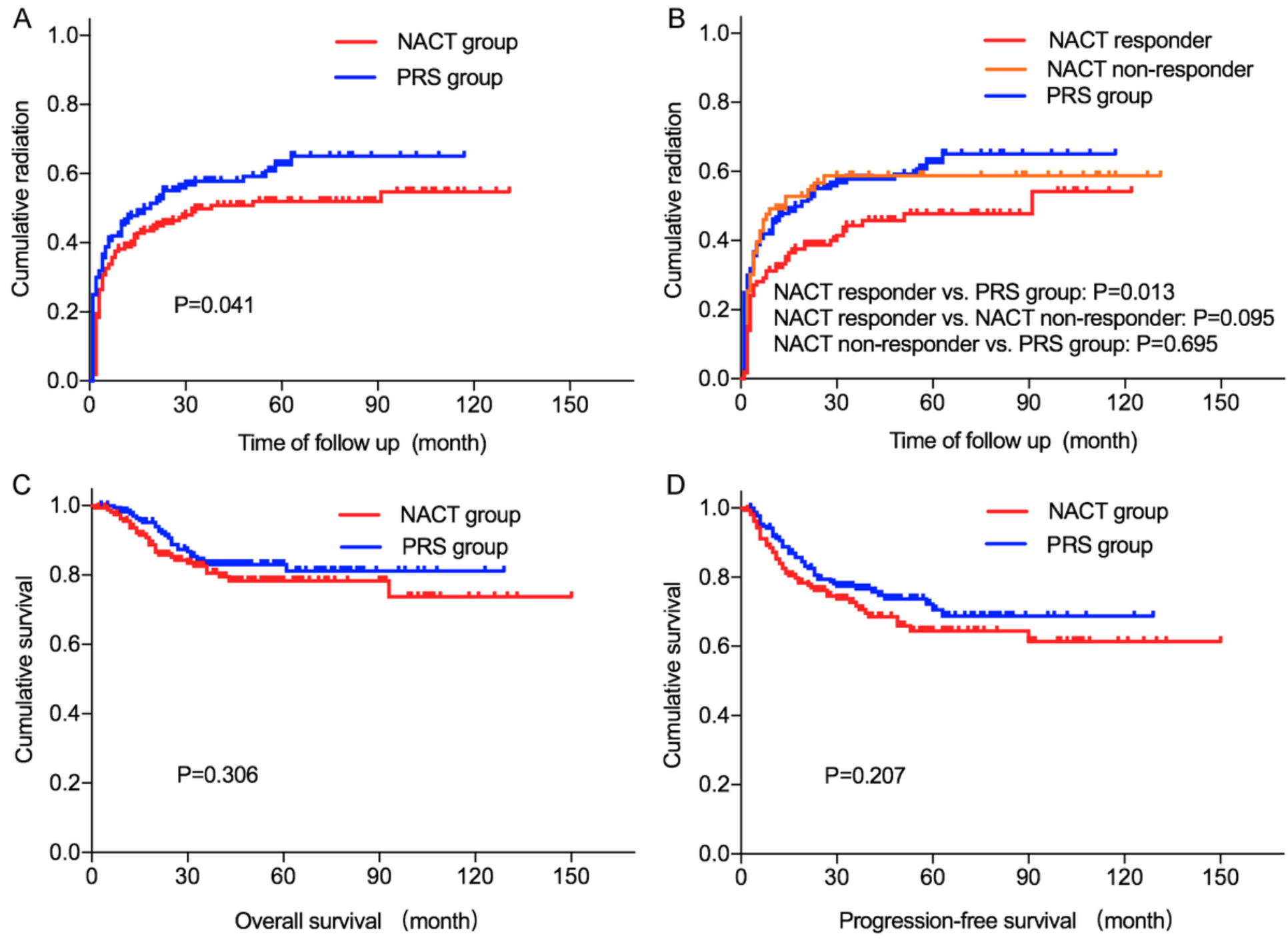

Figure 1 
Cumulative radiation rates and survival of different groups. The cumulative radiation rates of NACT group was significantly lower than that of PRS group (A). The cumulative radiation rate of NACT responders was significantly lower than PRS group, however with no significant difference between NACT non-responders and PRS groups (B). There was no significant difference in overall survival (C) and progression-free survival (D) between NACT and PRS groups. NACT, neoadjuvant chemotherapy; PRS, primary radical surgery. 